(C) 2009 IEEE. Personal use of this material is permitted. Permission from IEEE must be obtained for all other uses, in any current or future media, including reprinting/republishing this material for advertising or promotional purposes, creating new collective works, for resale or redistribution to servers or lists, or reuse of any copyrighted component of this work in other works 


\section{Segmentation and tracking of individual Pseudomonas aeruginosa bacteria in dense populations of motile cells}

\author{
Pascal Vallotton, Changming Sun, Dadong Wang \\ Division of Mathematical and Information Sciences \\ CSIRO \\ North Ryde, Australia \\ pascal.vallotton@csiro.au
}

\author{
Lynne Turnbull, Cynthia Whitchurch \\ Institute for the Biotechnology of Infectious Diseases \\ University of Technology \\ Sydney, Australia \\ Prabhakar Ranganathan \\ Australia Department of Mechanical \& Aerospace \\ Engineering \\ Monash University \\ Clayton, Australia
}

\begin{abstract}
The dynamics of individual bacteria underlies the manifestation of complex multicellular behaviours such as biofilm development and colony expansion. High resolution movies of expanding bacterial colonies reveal intriguing patterns of cell motions. A quantitative understanding of the observed behaviour in relation to the bacteria's own motile apparatus and to hydrodynamic forces requires that bacteria be identified and tracked over time. This represents a demanding undertaking as their size is close to the diffraction limit; they are very close to each other; and a typical image may contain over a thousand cells. Here, we describe the approach that we have developed to segment individual bacteria and track them in high resolution phase contrast microscopy movies. We report that over $99 \%$ of nonoverlapping bacteria could be segmented correctly using mathematical morphology, and we present preliminary results that exploit this new capability.
\end{abstract}

Keyword: bacteria, tracking, image analysis, image segmentation, cell motility, twitching motility, agar plate.

\section{INTRODUCTION}

As bacteria colonise surfaces, they can produce dramatic patterns of collective behavior that have fascinated scientists for centuries. Pseudomonas aeruginosa is an important opportunistic pathogen that causes infections in individuals affected by cystic fibrosis or in immunocompromised patients such as those with AIDS or those who are undergoing cancer chemotherapy. P. aeruginosa has evolved highly successful strategies for colonizing tissue surfaces, one of the first steps in the infection process. We are using live-cell imaging techniques to study twitching motilitymediated colony expansion of $P$. aeruginosa at high spatial and temporal resolutions to reveal how cells behave under different environmental conditions and genetic mutation, and to determine how cellular behaviours correlate with phenomena such as extracellular DNA release. In order to gain insight into the dynamics of individual bacteria during multicellular behaviors and to analyze the role of cell cell communication, chemosensing and hydrodynamics forces, a system that delivers the trajectories and morphological characteristics of individual bacteria over time is necessary. Such a system has been described earlier in the literature under conditions where sparse image fields of bacteria were investigated [1]. During colony expansion on agar, in contrast, the bacteria tend to lie so close to each other that they almost form a continuous sheet (see Fig. 1, inset). Examination of high resolution phase contrast microscopy images indicated that it is possible, at least visually, to recognize most bacteria in the images. One can expect the dynamics of adjacent bacteria to be correlated and contain information on cell-cell interactions. Such information would most likely be lost by the use of sparse sampling methods where only a small fraction of the bacteria are monitored (this can be achieved using fluorescence labeling of a subpopulation, for example). Thus we set out to solve the task of automatically segmenting and tracking every bacterium in phase contrast images. Our results show that it is possible to identify a majority of bacteria and reliably track them over time. Our system is expected to be useful to study complex multicellular bacterial behaviours such as biofilm development and colony expansion via twitching motility and swarming motility.

\section{METHODS AND RESUlTS}

\section{A. Sample preparation and image acquisition}

Microscopy of sub-surface colony expansion was performed using a $35 \mathrm{~mm}$ diameter Fluorodish petri dish (World Precision Instruments) containing $2.5 \mathrm{~mL}$ nutrient medium $(8 \mathrm{~g} / \mathrm{L}$ tryptone, $4 \mathrm{~g} / \mathrm{L}$ yeast extract, $4 \mathrm{~g} / \mathrm{L} \mathrm{NaCl})$ solidifed with GelGro (ICN) gellan gum $(8 \mathrm{~g} / \mathrm{L})$ instead of agar for greater optical clarity. The Fluorodish has a flat 
(0.17 mm thick) optical quality glass bottom, allowing the use of a short objective working distance, large numerical aperture (NA), and a high magnification (up to 100x). Bacteria were stab-inoculated to the interface between the solidified nutrient media and the glass base using a pipette tip, and incubated for $4 \mathrm{hr}$ at $37^{\circ} \mathrm{C}$. Colony expansion at the interstitial surface was then observed through the bottom of the Fluorodish using phase contrast microscopy with an Olympus IX71 inverted microscope fitted with a 100x oil objective (1.4 NA) and a Solent incubator chamber to maintain temperature at $37^{\circ} \mathrm{C}$ during image acquisition. The large NA and high magnification objective enabled high resolution imaging of individual bacterial cells in the expanding colony at the interstitial interface. Under phase contrast, bacteria appeared dark on a light background, such that they can be distinguished from each other by eye (Fig.1, inset). Even minute thermal drifts resulted in loss of focus. Therefore, refocusing had to be performed occasionally during acquisition. Typically, the experiments lasted about 1 hour, producing a few hundreds frames each. Images were $1376 \times 1032,8$ bit. Pixel size in the object space was equal to $64.5 \mathrm{~nm}$ and inter-frame time was equal to $5 \mathrm{~s}$.

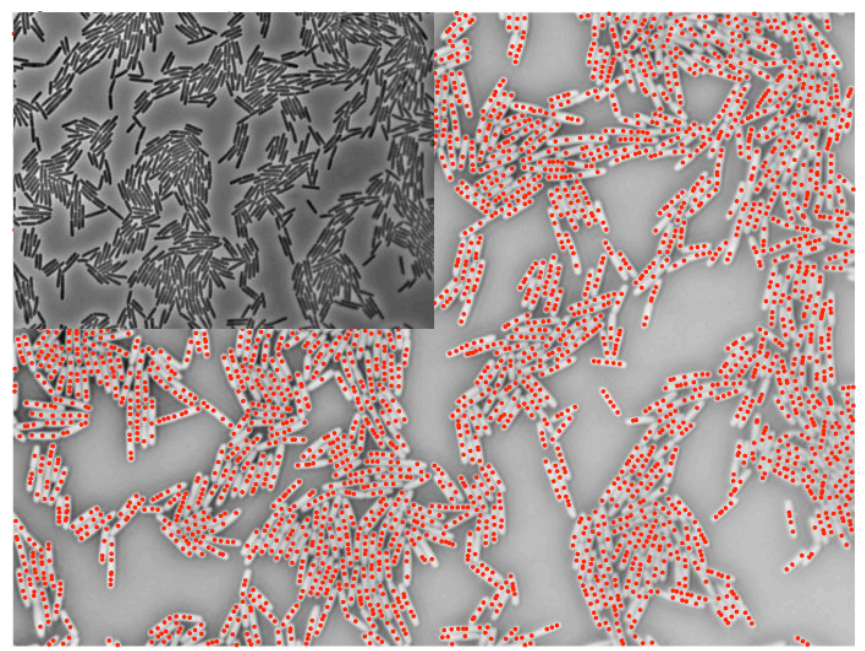

Fig. 1. Inset: Original phase contrast image of cells in a Pseudomonas aeruginosa interstitial colony acquired with a $100 x$ oil (1.4 NA) objective. The figure shows the original data after contrast inversion and Gaussian filtering. Local intensity maximum selection as described in the text produces robust markers associated exclusively with the bacteria

\section{B. Image analysis}

As the bacteria appeared dark on a light background, the images were first inverted: $\mathrm{I}=256-\mathrm{I}$. Due to minor inhomogeneities in the background and to the close proximity of bacteria, it is not possible to segment them using an intensity threshold, even a local one (results not shown). However, we found that we could generate robust markers associated with every cell as described next.

\section{1) Marker generation}

The images were filtered using a Gaussian convolution filter of width equal to 1 pixel. Local intensity maxima were then produced using the Matlab ${ }^{\mathrm{TM}}$ function "locmax $2 \mathrm{~d}$ " with an operator window of size $5 \times 5$ pixels. We then retained only those local maxima that presented a contrast value above 1.02 , as measured by dividing the intensity at the local maximum by the average intensity in a $5 \times 5$ window centered on that pixel. This created robust markers, such that every cell was associated with at least one marker, and no marker was present in the background (Fig. 1).

\section{2) Connected region generation}

A popular segmentation tool when robust seeds can be identified is the watershed transform [2]. However, the generation of watersheds is liable to leakage problems where the segmented regions spread beyond the true object boundaries. Another issue is that the watershed requires a single marker per cell. Improvements to the original watershed transform have been reported that deal with some aspects of these problems [3]. Rather than follow this path, we developed an alternative approach that, we will show, produces very few errors. We noted that line-like features separate adjoining bacteria. These linear features can be detected as described next and they are central to our methodology.

\section{3) Linear feature detection}

Our linear feature detector [4] detects ridge-like patterns in images by sampling the image intensity in multiple directions on short digital line segments (we typically use 8 so-called linear windows, each spanning 9 pixels). This process is repeated at every pixel position and only those pixels that represent a maximum intensity among all pixels in the linear window are kept in the result image. Further processing allows suppressing spurious features from the map. For example, we removed all connected components that were less than 7 pixels in diameter. We refer the reader to [4] and [5] for a more comprehensive description of this linear feature detection method.

Using linear feature detection only, traces are not generated at the interface between cells and the background. Thus we added (logical OR) to our linear feature map an edge map created using Canny edge filtering with default parameters (function "edge" under Matlab ${ }^{\mathrm{TM}}$ ) .

\section{4) Coarse region reconstruction}

The image resulting from the combined linear feature/edge detector contained connected regions, many of which were not associated with a bacterium. Thus, we used the seeds identified previously to retain only those connected regions associated with a bacterium using morphological reconstruction by dilation from the seeds [2]. This resulted in a decent segmentation, with over $97 \%$ of 1017 bacteria in total segmented satisfactorily (Fig. 2). In this manuscript, we do not attempt to split bacteria that are in the process of cell division. The latter can be recognized by a subtle constriction at the putative site of division, associated with a small intensity gradient, and often, a small kink. We operationally defined a bacterium to be undivided if the gradient between the two daughter cells was not sufficient to give rise to two segments using our method. This did not affect the tracking results significantly because the splitting outcome tended to be consistent from one frame to the next. In future work, we 
will develop our methodology further to better match the scoring that a microbiologist would obtain.

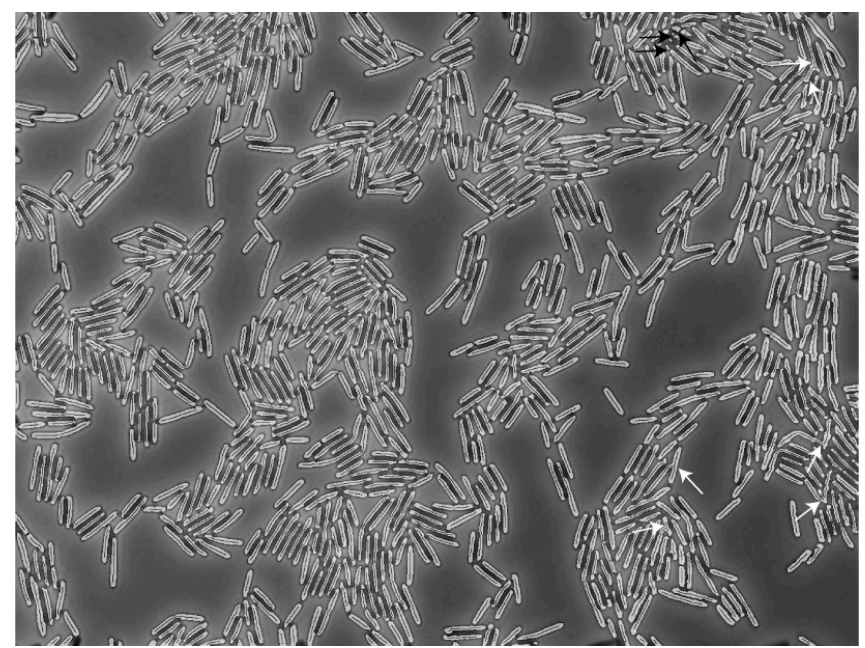

Fig. 2. Intermediate segmentation stage resulting from morphological reconstruction (details in text). A great majority of bacteria are segmented correctly. Errors are mostly of two types: - minor errors whereby the method fails to break a dividing bacteria at the putative break point - and topological errors (white arrows), which are more complex. We only deal with the latter type in this manuscript.

\section{5) Requirements on segmentation quality}

The error rate when tracking objects in a sequence of $\mathrm{M}$ frames assuming a segmentation error rate equal to $E$ ( $E$ was equal to 0.03 in our case) goes approximately as 1-((1$\left.\mathrm{E})^{\wedge} \mathrm{M}\right)$. This analysis does not take into account correlations effects that may improve the situation (it is often the same object that is repeatedly segmented inappropriately in multiple frames). Nor does our analysis account for tracking errors associated with the matching of objects that are correctly segmented yet tracked inappropriately (this makes the situation worse...). Using the expression above with a segmentation error rate equal to $3 \%$ and 100 frames in total, the fraction of correct tracks is expected to be only 5\%! It appears that more efforts need to be devoted to segmentation in order to generate a large proportion of long, correct trajectories.

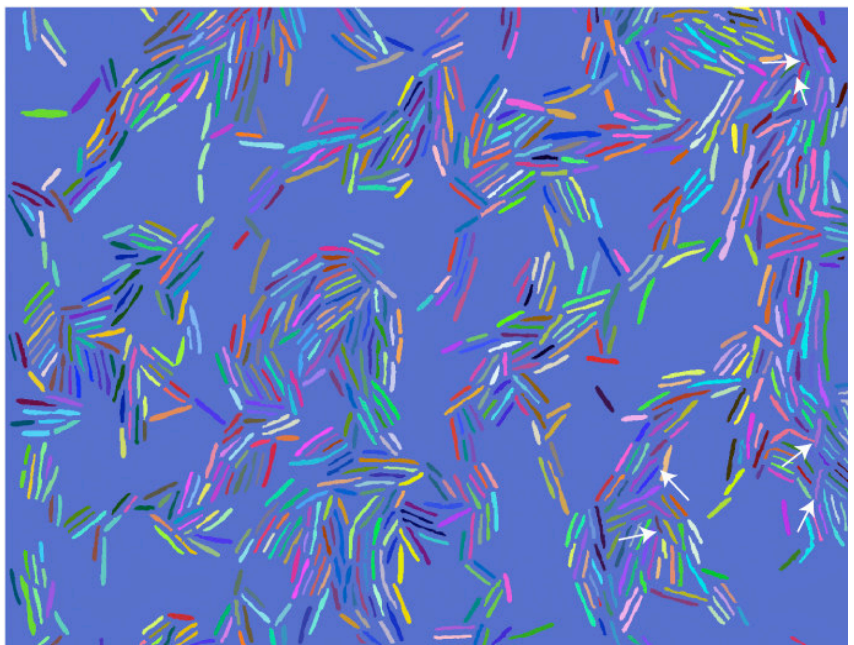

Fig. 3. Final segmentation. The white arrows highlight areas where segmentation errors where removed compared to Fig. 2.

6) Splitting of adjacent cells

Close examination of Fig. 2 reveals that most segmentation errors occur because adjacent bacteria were fused erroneously to each other. This happened because the cells were very close to each other, limiting the image gradient between them. In a majority of cases, the orientations of the fused bacteria in a particular cluster were different. In order to take advantage of this fact, we started by skeletonizing the connected regions to produce a medial axis along the bacteria, and we pruned small lateral branches that came up in the skeleton. Then, we identified all crossing points in the skeleton by using the fact that crossing points are the only skeleton points that possess more than 2 neighbors [4].

The skeleton was split at crossing points by removing from it the set formed by a dilation of the crossing points by a digital disk structuring element of radius 6 . This procedure ensures that the bacteria are modeled as independent straight rods, a picture that does not allow for crossing points. At that stage, all connected components in the skeleton that were entirely contained in the dilated set just described were removed. This ensured that only significant components were considered for the skeleton repair process that we describe next.

7) Repairing the skeleton

At this stage, the skeleton at crossing points has been split, such that two different bacteria abutting each other will not be fused in the final results. However, this process also disrupts the continuity of the skeleton along some legitimate bacteria. As bacteria are mostly rectilinear, the angle formed by different segments belonging to the same bacteria should be small. Thus, we linked together by digital lines all components that were forming an angle below 40 degrees. 


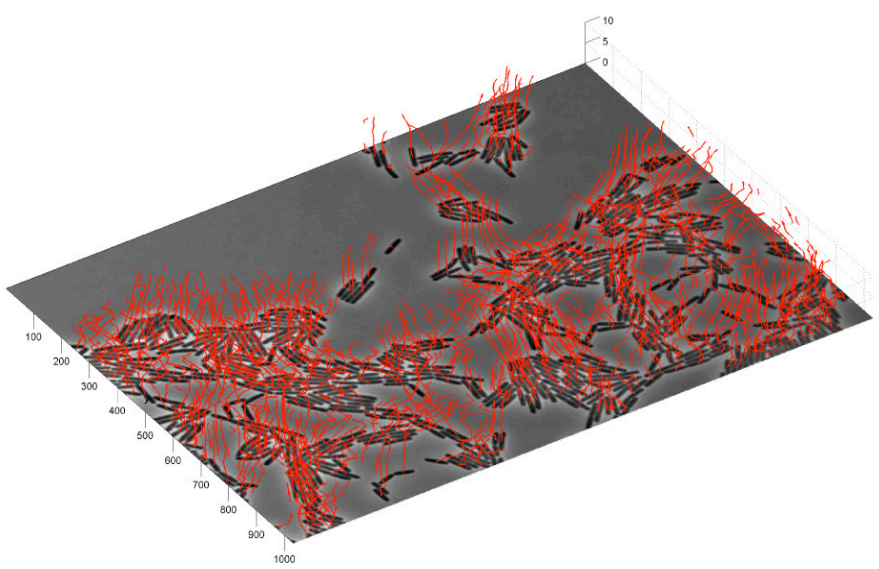

Fig. 4. First image frame of the sequence, overlaid with the trajectories of the centre of mass of the segmented bacteria.

\section{8) Refined reconstruction of bacteria}

A medial axis provides a satisfactory description of bacteria for many applications. However, if one is interested in the evolution of the size or shape of bacteria, one is also required to obtain the cell's boundaries. Thus, we used the skeleton as a seed for a watershed transform on the image gradient. Minima imposition [2] was performed using both the medial skeleton and the edge/linear feature mask (c.f. 3)). In exceptional cases, the skeleton was touching that mask with adverse effects on the result (the whole bacterium disappeared). The points of contact were removed in a straightforward manner prior to performing the watershed. The final segmentation is shown in Fig. 3, where black arrows highlight areas where segmentation mistakes were removed compared to Fig. 2. With the improvements described, bacteria were almost segmented stoichiometrically (>99\%).

\section{9) Object tracking}

Tracking bacteria individually is challenging. The amplitude of bacterial motion between each frame of our sequence is relatively large (up to 35 pixels) compared to the average distance between bacteria. This difficulty is made worse by the large aspect ratio of bacteria, which means that a few pixels only may separate the centre of mass of one bacterium from another when they lie side by side, as is often the case (Fig. 1). Our approach to object tracking aims to resolve such difficulties by the use of mathematical graphs that deals with the matching problem globally. This framework takes advantage of the knowledge that the number of objects is mostly conserved between frames. However, it is also flexible enough to allow for changes in the number of objects when there is enough evidence for such a possibility. As the methodology has been described elsewhere [6], details will not be reproduced.

Fig. 4 shows the trajectories of all bacteria in the field of view, with the time represented as the vertical axis. As can be observed, few trajectories only are broken - an indication that segmentation and tracking was satisfactory. Frame by frame examination of the movie, with the tracks overlaid onto the images confirmed the reliability of tracking. In Fig.
4, one notices groups of trajectories running almost parallel to each other. This corresponds to groups of cells moving in unison, held together by weak adhesive forces.

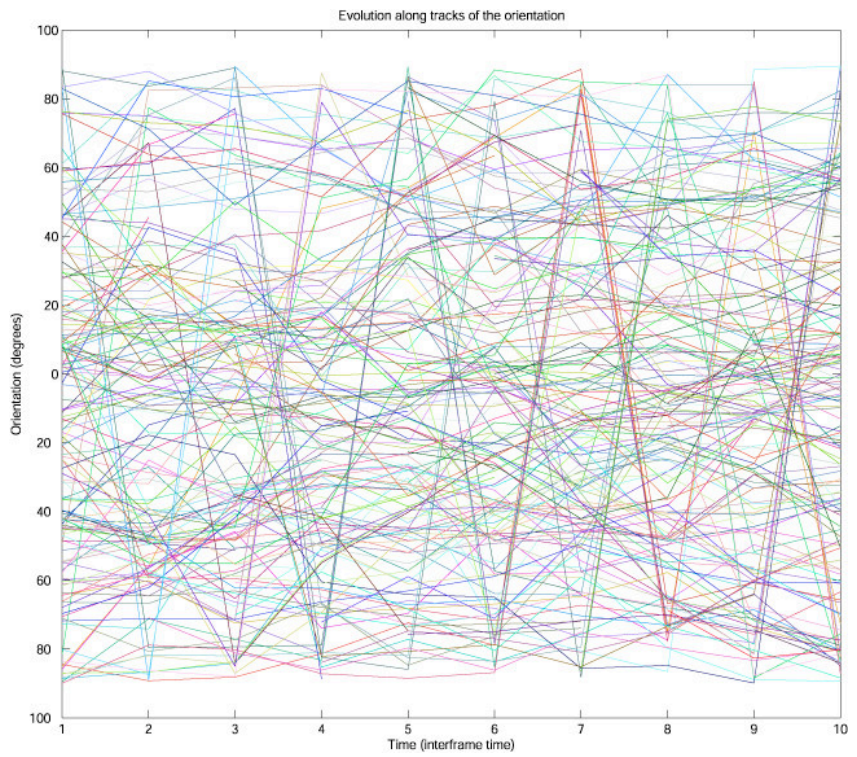

Fig. 5 Evolution of the orientation of the bacteria principal axis. Steep traces between -90 degrees and +90 degrees are due the equivalence of these two orientations

Fig. 5 represents the evolution of the orientation of the principal axis of bacteria. As the figure would be overcrowded otherwise, only tracks featuring an average velocity higher than 15 pixels/interframe time are shown. As the bacteria modify their orientation relatively slowly, most traces are relatively flat. The traces that cross the chart diagonally from the top to bottom and conversely are due to the equivalence between the +90 degrees orientation, and the -90 degrees orientation. The information contained in such graphs will help obtain a better understanding of the spatiotemporal characteristics of twitching motility.

\section{DISCUSSION AND CONCLUSIONS}

We only analysed 10 frames of the image sequence in this manuscript. In future experiments we will use a Nikon Ti inverted microscope with automated focus control, which will permit the acquisition of longer contiguous sequences of sharp images. Rare segmentation errors did subsist in regions where bacteria loose contact with the agar and overlap with each other. This tends to create strong image gradients within the bacteria and typically resulted in their fragmentation (Fig. 2 , black arrows). We are optimistic that this problem can be solved experimentally.

Our work mostly decouples the tasks of segmenting the bacteria from that of tracking them. We envision that we will be able to improve our results further by reexamining iteratively the segmentation results in the light of the tracking results, and inversely. Future improvements also include the refinement of the exact boundaries of cells, starting from the rough masks obtained in this manuscript. One should be able 
to exploit the well-conserved features of bacterial shape to that effect.

In summary, an automated system for the analysis of the motion and shape of individual bacteria in dense populations was presented. The method takes advantage of high resolution microscopy to distinguish bacteria less than 1 micrometer apart. A precise sequence of image analysis steps was defined that enabled excellent segmentation and produced trajectories mostly devoid of mistakes, as evaluated interactively. This new capability is expected to be fundamental for our future work, which will aim to understand how bacteria colonise surfaces efficiently. Future acquisition campaigns will involve hundreds of movies, each several gigabytes long. An important aspect will thus be to speed up the analysis, which presently takes 5 seconds per frame on an Intel core duo processor clocked at $2.6 \mathrm{GHz}$.

\section{ACKNOWLEDGMENT}

This work was funded by CSIRO and the Australian Research Council.

\section{REFERENCES}

[1] J. Xie, S. Khan, and M. Shah, "Automatic tracking of Escherichia coli bacteria," Med Image Comput Comput Assist Interv Int Conf Med Image Comput Comput Assist Interv., vol. 11, pp. 824-32., 2008.

[2] P. Soille, Morphological Image Analysis, 2 ed.: Springer, 2004.

[3] C. Vachier and F. Meyer, "The viscous watershed transform," Journal of Mathematical Imaging and Vision, vol. 22, pp. 251-267, May 2005.

[4] C. Sun and P. Vallotton, "Fast linear feature detection using multiple directional non-maximum suppression," J Microsc., vol. 234, pp. 147-57., 2009.

[5] P. Vallotton, R. Lagerstrom, C. Sun, M. Buckley, D. Wang, M. De Silva, S. S. Tan, and J. M. Gunnersen, "Automated analysis of neurite branching in cultured cortical neurons using HCA-Vision," Cytometry A., vol. 71, pp. 889-95., 2007.

[6] P. Vallotton, A. Ponti, C. M. Waterman-Storer, E. D. Salmon, and G. Danuser, "Recovery, visualization, and analysis of actin and tubulin polymer flow in live cells: a fluorescent speckle microscopy study," Biophys J., vol. 85, pp. 1289-306., 2003. 